\title{
Minimising delays to thrombolysis in patients developing acute myocardial infarction in hospital
}

\author{
Andrew D Mumford, Adrian P Banning
}

\begin{abstract}
Summary
We investigated the clinical features and management of 50 patients presenting with acute myocardial infarction from the community and 50 patients presenting with cardiac symptoms who developed an infarct after being admitted to a general medical ward for observation. Nineteen of the 50 patients initially admitted to nonspecialist wards were found retrospectively to have sustained an infarct prior to hospital admission. Of the remaining 31 patients in this group, 24 developed symptoms within 24 hours of admission, 26 presented with chest pain, while 21 had evidence of acute coronary ischaemia on the admission electrocardiograph. Of the 26 patients who presented with chest pain, 20 were treated with aspirin, 13 with intravenous nitrate and four with heparin. Median delay from onset of symptoms to thrombolysis with inhospital patients was 120 minutes and for community patients 287 minutes. Interestingly, the greatest component of this delay in both groups was the time taken for patients to decide to seek assistance after developing acute symptoms. Patients at high risk of developing acute myocardial infarction within 24 hours of hospital admission may be identified by a history of chest pain and electrocardiographic evidence of acute coronary ischaemia at admission. Such patients may experience suboptimal treatment and delays to thrombolysis if admitted to nonspecialist wards, but this may be reduced by admitting all 'high risk' patients directly to a high dependency cardiac ward for the first 24 hours after presentation.
\end{abstract}

Keywords: acute myocardial infarction, thrombolysis

Department of Cardiology,

University Hospital of Wales, Heath

Park, Cardiff CF4 4XN, Wales, UK AD Mumford

AP Banning

Correspondence to $\mathrm{Dr} \mathrm{AP}$ Banning, Department of Cardiology, John Radcliffe Hospital, Headington, Oxford OX3 9DU, UK

Accepted 12 December 1996
The delays which patients in the community experience prior to thrombolysis has attracted close scrutiny. Initiatives such as public education campaigns encouraging early presentation ${ }^{1}$ and 'fast track' admissions systems ${ }^{2}$ have been effective in reducing delays before administration of thrombolysis. However, not all patients develop symptoms of AMI in the community and audit of the admissions to our own coronary care unit demonstrated that up to $10 \%$ of our AMI patients developed AMI whilst already in hospital undergoing investigation or treatment for chest pain or unrelated complaints. Although it may be expected that their proximity to the coronary care unit would enable rapid diagnosis and treatment, our audit suggested that, paradoxically, some patients developing symptoms whilst already in hospital had longer delays to thrombolysis and an adverse medical outcome.

We prospectively collected data about delays prior to administration of thrombolysis in all patients admitted to the coronary care unit. Retrospective analysis of medical records was then used to determine details of treatment, monitoring and time delays involved in the management of 50 consecutive patients with AMI admitted from the general medical wards. The notes for 50 patients admitted directly to the coronary unit were also examined for comparison. Using these data we aimed to identify factors causing delays in the administration of thrombolysis and produce recommendations to improve patient care.

\section{Patients and methods}

The study was performed simultaneously in a large teaching hospital and a district general hospital in Wales. The existing admissions policies in these hospitals required that patients with acute chest pain were initially assessed in an admissions ward by the 'on-call' medical officer responsible for all emergency medical admissions. Patients were prioritised either for higher or lower dependency care according to their overall clinical condition and the appearance of the electrocardiograph (ECG). Patients with diagnosed AMI and most patients with unstable angina were transferred directly to the coronary care unit. Other patients with chest pain were transferred to general wards where a number of telemetric ECG monitors were available, allowing continuous 3-lead ECG monitoring; 12-lead ECG machines were available at all times on the general wards for patients with further symptoms after admission and there was a 'routine' 12-lead ECG service the morning after admission and on subsequent mornings. 
Fifty patients were identified who had originally been admitted to a general ward for observation of chest pain or related symptoms but who were subsequently diagnosed as having AMI and were transferred to the coronary care unit. In each case the diagnosis of AMI was confirmed by the presence of a compatible history, a two-fold rise in serum creatine kinase and characteristic ECG changes.

From retrospective analysis of the medical and nursing records of each patient we recorded demographic details, patient's previous experience and treatment of chest pain, and the nature and timing of patients' symptoms prior to admission. The initial diagnosis made by the 'on-call' medical officer at the initial assessment was recorded and this was compared to the diagnosis suggested by the admission ECG.

Once patients with chest pain had been admitted to the general wards their subsequent clinical course was documented, including the use of anti-anginal therapies, telemetric ECG monitoring, and analysis of 12-lead ECG records. Using descriptions of patients' symptoms recorded in the medical records, in conjunction with the appearance of ECGs performed on the wards, the timing of AMI was determined in each case. The time the clinical diagnosis of AMI was reached, times of arrival in the coronary care unit, and administration of thrombolysis were also determined.

A control group of $50 \mathrm{AMI}$ patients sustaining AMI in the community were recruited from direct admissions to the coronary care unit during the same period. All these patients satisfied the same diagnostic criteria for AMI as the study group. The timing of onset of symptoms, arrival at hospital, transfer to the coronary unit, and start of thrombolysis was recorded for each control patient. Blinded analysis of all ECGs in the study was performed by two independent observers. ECGs were categorised as either normal, previous AMI, acute cardiac ischaemia or AMI, according to previously documented criteria. $^{3}$

From the study start date in December 1994, 50 consecutive eligible patients were selected retrospectively. An additional five patients were excluded due to unavailability of documentation of clinical events. The study period covered an 18-month period and was therefore not significantly influenced by seasonal variations in admission practice. This represented a period of normal clinical activity during which more than 9000 emergency admission patients were assessed at both hospitals. Total admissions to the coronary care units in this period was approximately 1200 patients, including 520 with confirmed AMI. In view of the marked skewing of timings recorded in the study, median values and quartile ranges were calculated as measures of central tendency and spread. Statistical analysis of data was performed using MINITAB.

\section{Results}

Our patients consisted of 74 males and 26 females of mean age 69 years (range 37-93). Of these 100 patients, 36 described a previous history of AMI and 47 described previous angina; 42 patients had pre-existing prescriptions for aspirin, eight for a $\beta$-blocker and 82 for sublingual nitrate. There were no differences in the pre-admission characteristics of control and study patients. The median delays from onset of symptoms to diagnosis of AMI, subsequent transfer to the coronary care unit and administration of thrombolysis are shown in the table.

\section{CONTROL GROUP}

The greatest delay to treatment in the group admitted directly to the coronary care unit was from the onset of symptoms to the diagnosis of AMI. Median values and quartile ranges of the constituent delays were: delay in patients deciding to seek medical help after developing symptoms $98 \mathrm{~min}(20-222)$, delay in awaiting consultation with the general practitioner 40 min $(20-60)$, transport time to hospital $13 \mathrm{~min}(7-27)$, and delay in initial assessment on arrival at hospital prior to diagnosis $45 \mathrm{~min}$ $(30-110)$. Thrombolysis was administered to the majority ( $84 \%)$ of the 50 patients in the control group and was withheld because of a major contraindication in six $(12 \%)$ No reason for withholding thrombolysis was given in the remaining two patients. Six of the control patients died in the coronary care unit.

\section{STUDY GROUP}

Of the 50 patients who were initially admitted to a general ward, $19(38 \%)$ were found to have sustained a recent AMI in the community prior to arrival at hospital. These included 14 inferior and five anterior territory infarctions. These patients presented with chest pain and were transferred to a general ward after receiving initially incorrect diagnoses from the admitting medical officer (angina in 13 cases and noncardiac chest pain in six). Subsequent transfer to the coronary care unit was initiated

Table Median delays and quartile ranges ( $\mathrm{min}$ ) in patients suffering AMI in hospital or in the community

\begin{tabular}{lcc}
\hline Delay & In-hospital patients & Control patients \\
\hline Onset of symptoms to diagnosis of AMI (min) & $55(30-120)$ & $195(84-268)$ \\
& $n=31$ & $n=50$ \\
Transfer time to coronary unit after diagnosis of AMI (min) & $50(25-85)$ & $n=31$ \\
Onset of symptoms to thrombolysis (min) & $120(85-180)$ & $n=50$ \\
& $n=22$ & $287(62-390)$ \\
& & $n=42$ \\
\hline
\end{tabular}


after 'routine' review of the patients by senior medical staff in 14 of these 19 cases. In the remaining five patients, urgent or emergency review by senior staff was requested because of rapid clinical deterioration. This included three patients who developed ventricular fibrillation on the ward without complaining of further chest pain or having a second ECG after admission. The median delay from arrival in the emergency admissions ward to reaching the correct diagnosis in this group was 330 minutes (quartile range $75-2040$ ) and median delay in their subsequent transfer to the coronary unit was 18 minutes (quartile range $8-45)$. Ten of the $19(53 \%)$ patients who had sustained AMI before admission subsequently received thrombolysis. A major contraindication prevented thrombolysis in three $(16 \%)$ cases but in the remaining six cases no reason was given for omission of treatment. Four of the 19 patients in this group subsequently died in the coronary care unit.

The remaining 31 study patients had no evidence of AMI at admission but developed AMI sometime after admission to the general medical wards. The majority $(84 \%)$ of these patients were originally admitted to hospital with chest pain, while two patients presented with dyspnoea, two with abdominal pain, and one with collapse. Acute coronary ischaemia was evident on the ECG performed at the initial assessment in the admissions ward for 21 $(68 \%)$ of these patients. Of the remainder, five patients showed evidence of previous AMI. After scrupulous examination, no ECG abnormality could be found in the remaining five patients. Monitoring and treatment of patients admitted initially to the general medical wards, both with misdiagnosed AMI and with unstable angina, is shown in figure 1 .

The median interval from original hospital admission to development of the symptoms of AMI on a general ward was 420 minutes (quartile range $165-2520)$ and $24(77 \%)$ patients developed AMI within 24 hours of

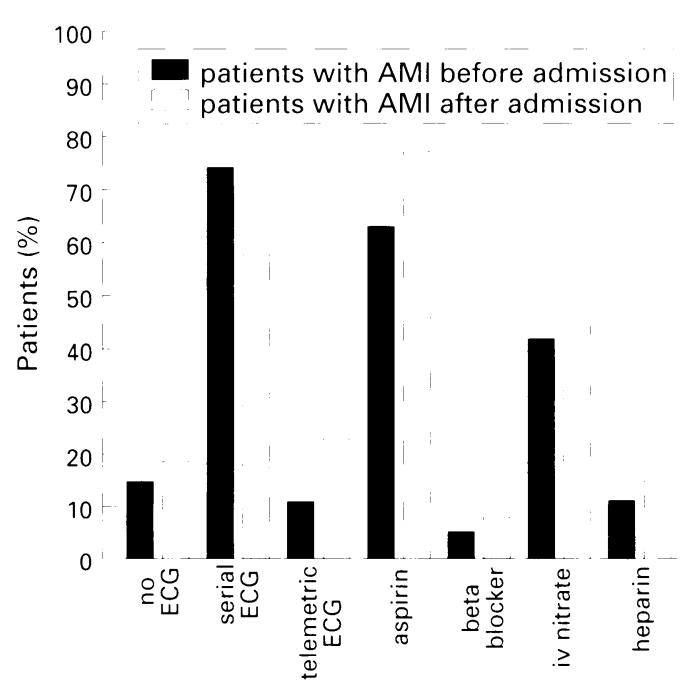

Figure 1 Monitoring and treatment of chest pain in patients who sustained AMI before admission $(n=19)$ and patients developing AMI after admission $(n=26)$ to the general wards admission (figure 2). The largest component of the delay between onset of symptoms and thrombolysis in the study group was delay awaiting assessment and diagnosis of AMI by the 'on-call' medical officer.

The pattern of delays in diagnosis for patients developing AMI on the ward is shown in figure 3. Twelve patients waited for more than an hour after developing symptoms before receiving a diagnosis of AMI. Four patients developed acute symptoms of chest pain during the night but did not alert the nursing staff, including one patient receiving continuous monitoring with telemetric ECG recording. In all four cases, the diagnosis of AMI was made only after inspection of the 'routine' morning ECG, resulting in delays to thrombolysis of three to eight hours. Another four patients reported their symptoms promptly but

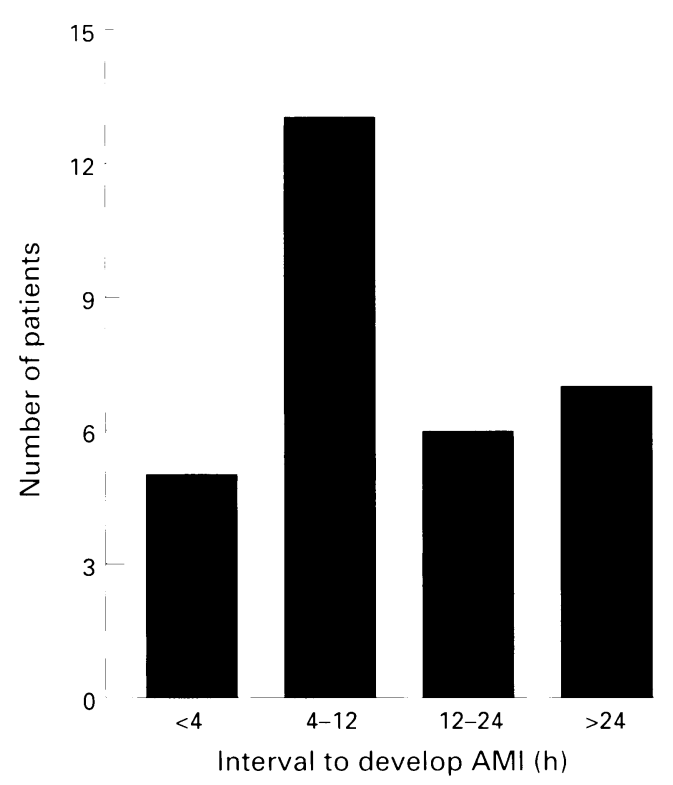

Figure 2 Interval between admission to hospital and development of AMI on the general medical wards

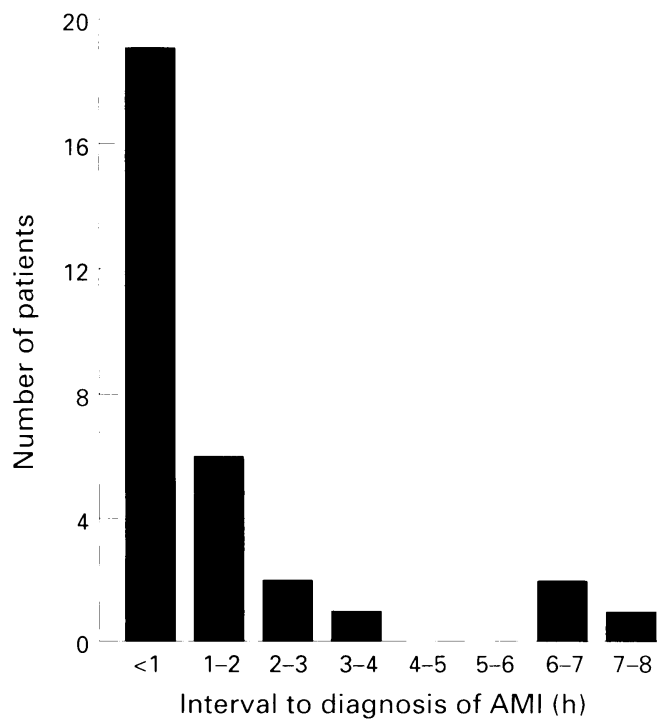

Figure 3 Interval between onset of svmptoms and diagnosis for patients developing AMI on the general medical wards 
did not receive a diagnosis for more than an hour whilst the nursing staff administered antacids or sublingual nitrates prior to notifying the 'on-call' medical staff. A delay of more than an hour to diagnosis occurred in four patients awaiting medical review and ECG.

Thrombolysis was administered in 22 of the 31 patients who sustained AMI after admission. Treatment was withheld because of a major contraindication in four of these patients but in the remaining five no reason was recorded in the medical records. The inhospital mortality of patients sustaining AMI on the general wards was $16 \%$.

\section{Discussion}

This review of admissions to the coronary care unit raises important issues in the management of patients who present with chest pain and it has prompted important changes in our management of these patients.

For patients who develop AMI in the community, it has been suggested that at least $50 \%$ of eligible cases should receive thrombolysis within 90 minutes of the onset of major symptoms., ${ }^{4,5}$ Our data show that in patients who develop AMI on the general medical wards, thrombolysis is delayed beyond these recommendations, even though close supervision of patients in hospital should facilitate rapid diagnosis and treatment. These delays to thrombolysis reduce the chances of a favourable outcome after AMI.

For patients sustaining AMI in the community, rapid access to intensive monitoring and early thrombolysis can only occur if both patients and medical services follow an optimum course of action. Community patients must recognise the significance of their symptoms of chest pain and decide to seek medical assistance. The community medical services must then assess the patient, reach a diagnosis, and arrange transport to hospital. Delays to treatment can occur at any of these steps but the greatest delay occurs whilst patients decide to seek medical assistance after developing acute symptoms. ${ }^{1}$

This course is similar for patients who develop symptoms of AMI whilst in hospital. Patients must understand the significance of a further episode of chest pain, even though it may be similar in character to their original presenting symptoms. Patients must decide that assistance is required and then alert the medical staff, usually by contacting one of the ward nurses. They in turn must contact the 'on-call' medical officer who is reponsible for assessing the patient and performing a diagnostic ECG. Once a diagnosis has been established, arrangements can be made to 'transport' the patient to the coronary care unit.

Our study shows that unnecessary delays in the treatment of patients developing AMI after admission occurred at all of these stages but that the longest delays were between the onset of symptoms and diagnosis of AMI. In common with patients who developed AMI in the community, the greatest component of this delay was the time taken for patients to decide to seek assistance after developing symptoms. The reasons for this are likely to be multifactorial but may have included the failure by patients to appreciate the significance of new symptoms of acute chest pain. This may have been particularly important in patients who originally presented with chest pain or those with long-standing experience of angina prior to admission. Patients may also have been reluctant to disturb nursing staff or other patients, particularly at night.

Once diagnosis had occurred, there was frequently delay in transferring patients from the general medical ward to the coronary care unit. As local guidelines prevented the administration of thrombolysis on general medical wards, delays to treatment frequently occurred while arranging bed space and awaiting porters. Excessive delays did not affect patients who were transferred to the coronary care unit directly from the emergency admissions ward.

The greatest delays in treatment occurred in patients who had sustained AMI in the community, but were first admitted to the general wards due to diagnostic error by the admitting medical officer. In each of these cases there were sufficient criteria on the admission ECG alone to diagnose AMI. However, correct diagnosis was only reached when the patient and their admission ECG were reviewed by an experienced staff member, although this was frequently some hours later. The median delay in diagnosis of 330 minutes in this group may have been substantially reduced by senior review of patients within the admissions ward. Diagnostic accuracy by junior medical staff in the admissions ward may have been improved by the emergency measurement of cardiac enzymes, particularly 'early enzymes' such as tropinin-I. There remains however, intrinsic delay in laboratory analysis and in all of the misdiagnosed patients in this study, there was sufficient information for diagnosis in the clinical history and ECG alone, both of which were available within minutes of arrival of the patient in hospital.

The availability of national and local treatment protocols to junior medical staff has greatly simplified management of patients who present with AMI. However, the management objectives of patients who present with unstable angina, particularly in the first 24 hours of their admission, have been poorly defined.

Our study indicates that, with existing practices, patients admitted to the general wards with unstable angina may receive suboptimal care. These patients are in danger of developing AMI yet, if they are admitted to the general wards, continuous ECG monitoring is frequently unavailable and nursing staff may not be familiar with the symptoms and signs of impending AMI. There also appears to be reluctance by 'on-call' medical staff to use standard anti-anginal therapies that would be regarded as routine for patients admitted to the coronary care unit. This is consistent with 
previous observations that patients admitted to nonspecialist wards received intensive antianginal therapy less often and had poorer outcomes than patients admitted to a dedicated cardiac ward. ${ }^{6}$

Our observation that $68 \%$ of the patients who sustained AMI after admission had evidence of acute coronary ischaemia on admission is an important finding. It demonstrates that, in most cases, accurate interpretation of the admission ECG allows the identification of a large proportion of patients who are at risk of developing AMI. However, it must be remembered that a normal resting ECG does not exclude significant underlying coronary artery disease. ${ }^{7}$ A presenting complaint of chest pain was present in $84 \%$ of our patients, indicating that this is a more sensitive indicator of impending AMI, although clearly less specific. Chest pain is amongst the commonest presenting symptoms of patients assessed on a general medical 'take'. To advocate intensive cardiac monitoring of all these patients throughout their hospital admissions would have dramatic financial implications. Our finding that $77 \%$ of patients who developed AMI on the general wards did so within a day of admission suggests that patients may be considered at particular risk within 24 hours of an acute episode of chest pain. We suggest that it is only during this brief period

1 Herlitz J, Hartford M, Blohm M, et al. Effect of a media campaign on delay times and ambulance use in suspected acute myocardial infarction. Am 7 Cardiol 1989; 64: 90-3. 2 Pell ACH, Miller HC, Robertson CE, Fox KAA. Effect of 'fast-track' admission for acute myocardial infarction on delay to thrombolysis. $B M \mathcal{F}$ 1992; 304: 83-7.

3 ISIS-2 Collaborative Group. Randomised trial of intravenous streptokinase, oral aspirin, both or neither among 17,187 cases of suspected myocardial infarction: ISIS-2. 17,187 cases of suspected

4 DeBono DP, Hopkins A. The management of acute myocardial infarction: guidelines and audit standards. Report of a workshop of the joint audit committee of the British Cardiac Society and the Royal College of Physicians. f $R$ Coll Physicians London 1994; 28: 312-7.

\section{Summary/learning points}

- careful analysis of the ECG on admission can help risk-stratify patients presenting with chest pain

- patients who present with chest pain and ECG evidence of ischaemia are at a high risk of further cardiac events and require careful assessment, treatment and monitoring

- patients who experience myocardial infarction whilst in hospital have a high mortality rate and require urgent medical attention

that intensive cardiac monitoring should be available.

Following the completion of this study, we have modified our admissions policy for patients presenting with chest pain. All patients are reviewed at admission by a physician of at least senior house officer grade. All patients with ECG changes suggestive of acute cardiac ischaemia or with a suggestive history are now admitted to the coronary care unit or to a newly opened cardiac high-dependency ward. Observation here is for at least 24 hours. We believe that these changes have improved our standard of care and will result in a concomitant reduction in mortality reflecting earlier diagnosis and earlier access to appropriate management.

5 Lawson-Matthew PJ, Wilson AT, Woodmansey PA, Channer KS. Unsatisfactory management of patients with myocardial infarction admitted to general medical wards. $\mathcal{f}$ $R$ Coll Physicians London 1994; 28: 49-51.

6 Schreiber TL, Elkhatib A, Grines C, O'Neil WW. Cardiologist versus internist management of patients with unstable angina: treatment patterns and outcomes. $¥ A C C$ 1995; 26: angina: treat

7 Norell M, Lythall D, Coghlan G, et al. Limited value of the resting electrocardiogram in assessing patients with recent onset chest pain: lessons from a chest pain clinic. Br Heart $\mathcal{F}$ 1992; 67: 53-6. 